considered, ${ }^{1}$ but the patient showed no radiological or bone-scan abnormalities and the complete and rapid remission was contrary to such a diagnosis. Peripheral nerve abnormality suggested by hyperaesthesia was corroborated at necropsy by the presence of haemosiderin in the perineurium and epineurium. Haemosiderin deposition in nerves at similar sites has been described in haemochromatosis, ${ }^{2}$ but this patient did not have cirrhosis or skin pigmentation.

Haemorrhage-induced neuropathy is well recognised in haemophilia ${ }^{3}$ but is not a characteristic finding in aplastic anaemia. Intraneural haematoma has been described after trauma ${ }^{4}$ and in patients receiving oral anticoagulants and heparin. ${ }^{3}$ Bigelow and Graves $^{5}$ reported intraneural haemorrhage due to toxic thrombocytopenic purpura caused by penicillin. Experimental work has shown that haemorrhage into nerves can produce a similar picture, with no residual functional loss when the blood is resorbed. ${ }^{5}$

We suggest that the bizarre clinical presentation of intractable pain with hyperaesthesia was caused by periodic haemorrhage into peripheral nerves of the legs and lumbar plexus. The importance of HLA-matched platelet transfusion as supportive treatment in aplastic anaemia is emphasised by this report.

${ }^{1}$ Bain B. Extensive bone marrow infarction followed by myelofibrosis in a patient with $\mathrm{Ph}^{1}$ positive chronic granulocytic leukaemia. $\mathcal{F}$ Clin Pathol $1980 ; 33: 449-53$.

${ }^{2}$ Melnick SC, Whitfield AG. Polyneuritis in haemochromatosis. Postgrad Med F $1962 ; 38: 580-3$.

${ }^{3}$ Patten BM. Neuropathy induced by haemorrhage. Arch Neurol 1969; $21: 381-6$.

${ }^{4}$ Richardson RR, Hahn YS, Siqueira EB. Intraneural haematoma of the sciatic nerve. $\mathcal{F}$ Neurosurg $1978 ; 49: 298-300$.

${ }^{5}$ Bigelow NH, Graves RW. Peripheral nerve lesions in haemorrhagic diseases. Archives of Neurology and Psychiatry 1952;68:819-30.

(Accepted 10 March 1981)

University Departments of Haematology and Pathology, Institute of Pathology, Royal Victoria Hospital, Belfast BT12 6BA

C, BHARUCHA, MB, MRCPATH, senior registrar in haematology

$\mathrm{J}$ CROSBIE, MB, registrar in pathology

H BHARUCHA, MD, MRCPATH, consultant pathologist

E E MAYNE, MD, MRCPATH, consultant haematologist

\section{Cardioversion 125 times without necrosis}

Evidence exists that repeated electrical cardioversion is associated with cardiac necrosis, ${ }^{1-3}$ and this knowledge may result in a reluctance to persist with cardioversion for fear of leaving a surviving patient with severely impaired cardiac function. We report on a patient given repeated cardioconversion in whom there was no clinical or electrocardiographic evidence of impaired cardiac function.

\section{Case report}

A 41-year-old woman was admitted to hospital after having taken 200 dothiepin $(25 \mathrm{mg})$ tablets in a suicide bid. After an initial period of stability she developed ventricular fibrillation, which responded to cardioversion ( 150 joules) but rapidly recurred. She was intubated and ventilated. After the fifth episode of ventricular fibrillation she was paralysed with alcuronium chloride and continuous positive-pressure ventilation with $40 \%$ oxygen was administered.

Over the next 18 hours ventricular fibrillation occurred over 125 times, on each occasion reverting to sinus rhythm after the application of cardioversion (50-150 joules). The rate of occurrence of ventricular fibrillation was uninfluenced by the administration of lignocaine, mexiletine, disopyramide, procainamide, magnesium sulphate, and phenytoin.

She made a good recovery without any clinical evidence of impaired cardiac function. Glutamine oxaloacetate transaminase activity rose to $333 \mathrm{IU} / \mathrm{l}$ (normal less than $42 \mathrm{IU} / \mathrm{l}$ ) and lactate dehydrogenase activity to $821 \mathrm{IU} / 1$ (normal less than $725 \mathrm{IU} / \mathrm{l}$ ). There were no electrocardiographic changes either immediately after recovery or on review two months later.

\section{Comment}

We believe in the light of this case that, when oxygenation is adequate and the myocardium healthy, repeated cardioversion in man may not be associated with such severe cardiac necrosis as previous reports have indicated. The possibility remains that the tricyclic antidepressant may have protected the myocardium against electrical injury. We know of no evidence, however, supporting this view.

${ }^{1}$ Dahl CF, Ewy GA, Warner ED, Thomas ED. Myocardial necrosis from direct current countershock. Circulation 1974;50:956-61.

2 Warner ED, Dahl C, Ewy GA. Myocardial injury from transthoracic defibrillator countershock. Archives of Pathology 1975;99:55-9.

${ }^{3}$ Van Vleet JF, Thacker WA Jr, Geddes LA, Ferrans VJ. Acute cardiac damage in dogs given multiple transthoracic shocks with a trapezoid wave generator. Am $\mathcal{F}$ Vet Res 1977;38:617.

(Accepted 9 March 1981)

Clinical Research Unit, Hallamshire Hospital, Sheffield S10 2JF PAUL A CANN, MRCP, research fellow

District Hospital, York YO3 7HE

CHARLES F CORKE, MRCP, DA, medical registrar

\section{Recurrent bacteraemia: an unusual presentation of choriocarcinoma}

Choriocarcinoma is an uncommon neoplasm that may present in unusual ways. We report on a patient with a choriocarcinoma who presented with three episodes of bacteraemia in seven weeks after the premature delivery of a normal child.

\section{Case report}

At 34 weeks of a previously uneventful pregnancy a healthy 28 -year-old gravida 2 delivered a live female infant after an antepartum haemorrhage. She was admitted to this hospital 19 days post partum with a five-day history of fever and rigors. There was no vaginal discharge.

On admission she was febrile, and the spleen was palpable $2 \mathrm{~cm}$ below the costal margin. Six blood cultures were taken; Bacteroides fragilis was isolated from four, and Escherichia coli from the two others. Urine was sterile; $E$ coli was grown from a high vaginal swab. She was treated with metronidazole and co-trimoxazole for two weeks. Her fever settled rapidly, and the spleen was not palpable on discharge from hospital.

Seven days after finishing the antibiotics she developed fever and rigors, having had vaginal bleeding for three days. On readmission she was ill and febrile with a palpable spleen. A bulky uterus was felt on vaginal examination. Five blood cultures grew $E$ coli. She was treated with gentamicin and the fever settled rapidly. Four days later bulky material was evacuated from the uterus. Histologically this was trophoblastic and deciduous tissue. $E$ coli was isolated on culture.

After the evacuation she became hypotensive with fever, rigors, and heavy vaginal bleeding. Two blood cultures grew $E$ coli. She was treated with cefuroxime and by blood transfusion. Her condition improved, but for the next two weeks she remained unwell with intermittent fever and haemoglobin concentration fell to $7 \mathrm{~g} / \mathrm{dl}$. She developed vaginal bleeding, and the uterus was again found to be enlarged.

On the 64th postpartum day a further uterine evacuation was performed under co-trimoxazole prophylaxis. Histologically the material obtained showed active trophoblastic tissue with invasion of blood vessels in the myometrium. Urinary excretion of chorionic gonadotrophin after the evacuation was $54950 \mathrm{IU} / 24 \mathrm{~h}$. She was transferred to another hospital for chemotherapy. Nine months later she remained well, with no evidence of recurrent disease, and had had no further episodes of infection.

\section{Comment}

Bacteraemia is a rare complication of choriocarcinoma. It has been reported in a patient receiving chemotherapy ${ }^{1}$ but not as the presenting complaint. Although the tumour is prone to necrosis, infection appears to be rare. One patient is recorded who presented with two episodes of "septic abortion" in two weeks, ${ }^{2}$ and fever and a foul discharge are mentioned as features of advanced disease in a gynaecological textbook. ${ }^{3}$ We presume that the episodes of bacteraemia in this patien were secondary to infection of the intrauterine tumour; at no time was there neutrophil leucocytosis, but the absence of previous problems with infection suggests that she did not have an immune deficiency predisposing to infection.

This case shows how choriocarcinoma may present to genera 
physicians, with little to suggest the underlying disease, so that the diagnosis of choriocarcinoma may not be considered for some time.

We thank Mr T F Redman for gynaecological advice and comments on this report; Dr G W Pennington and Dr F E Neal for chemotherapy and subsequent care of the patient; and the Public Health Laboratory, Leeds, for bacteriological studies.

${ }^{1}$ Lacey CG, Futoran R, Morrow CP. Clostridium perfringens infection complicating chemotherapy for choriocarcinoma. Obstet Gynecol 1976; 47:337-41.

2 Donald I. Practical obstetric problems. 5th ed. London: Lloyd-Luke, 1979.

${ }^{3}$ Jeffcoate N. Principles of gynaecology. 4th ed. London: Butterworths, 1975.

(Accepted 10 March 1981)

Department of Infectious Diseases, Seacroft Hospital, Leeds LS14 6UH

PAUL HOLT, MB, MRCP, senior registrar

ROY NICHOLAS, MD, MRCP, medical assistant

\section{Hypertrophic osteoarthropathy and purgative abuse}

We describe a young woman who presented with clubbing of digits and hypertrophic osteoarthropathy. A history of purgative abuse emerged and it appeared likely that this was more than a chance association. We are unaware of any previous reports linking hypertrophic osteoarthropathy with purgative abuse.

\section{Case report}

A 21-year-old dental nurse presented in mid-1978 with a nine-month history of painful swelling of both ankles followed by painful swelling and morning stiffness affecting proximal and distal interphalangeal joints of both hands. Her only other symptom was intermittent diarrhoea of three years duration. There was no family history of clubbing or any rheumatic disorder.

On examination she weighed $49.1 \mathrm{~kg}$. There was clubbing of fingers and toes with pronounced periungual erythema. Both ankles were swollen, red, and tender, and there was tenderness of interphalangeal joints of the fingers. General examination showed no other abnormality. Cardiovascular and respiratory systems were normal, as were the findings on abdominal and rectal examination. Results of sigmoidoscopy to $15 \mathrm{cms}$ were similarly completely normal.

Investigations showed an erythrocyte sedimentation rate of $10 \mathrm{~mm}$ in first hour, haemoglobin concentration $13.9 \mathrm{~g} / \mathrm{dl}$, and white cell count $8.3 \times$ $10^{9} / 1\left(8300 / \mathrm{mm}^{3}\right)$. Serum electrolyte, urea, and protein concentrations were normal, as were iron and folate. Serum calcium concentration was 2.5 $\mathrm{mmol} / \mathrm{l}(10.0 \mathrm{mg} / 100 \mathrm{ml})$, phosphate concentration $1.1 \mathrm{mmol} / 1(3.5 \mathrm{mg} / 100$ $\mathrm{ml}$ ), and alkaline phosphatase activity $114 \mathrm{u} / \mathrm{l}$ (normal range 20-90). Autoantibody screen was negative and thyroid function values normal. Radio-

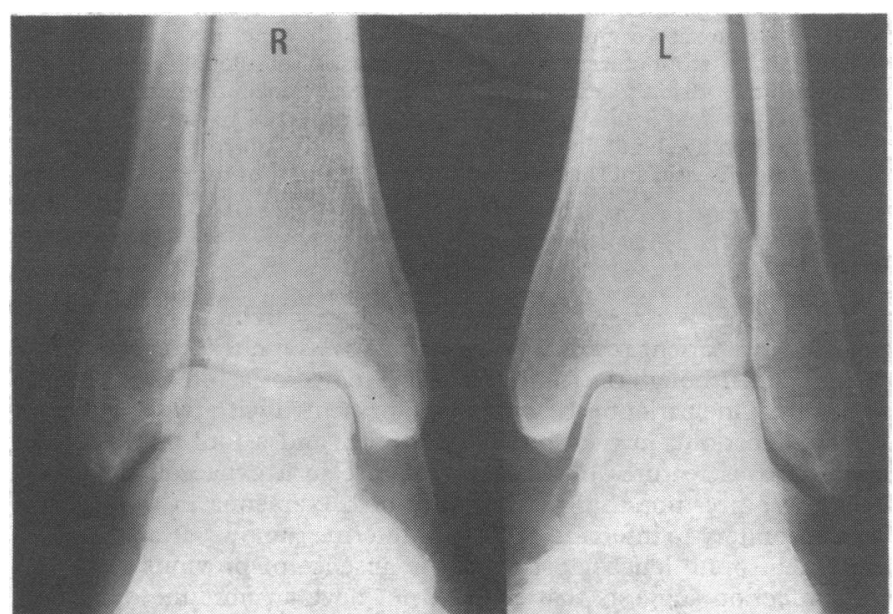

Radiograph of ankles showing normal joints but florid periosteal reaction affecting distal tibiae. graphs of chest and hands were normal but those of knees and ankles showed striking symmetrical bilateral periosteal new bone formation, affecting particularly the ends of the long bones (figure). Barium follow-through and enema examinations showed only mild flocculation of contrast material in the small bowel.

The patient's mother disclosed that for three years the patient had been taking at least three senna (Senokot) tablets daily. The patient then confessed to habitually taking this aperient to control her weight. She also admitted to a period of secondary amenorrhoea of several months' duration a year before.

In October 1978 she stopped taking laxatives, and subsequently her weight increased to $57.2 \mathrm{~kg}$. Within six months the clubbing had disappeared, though the periungual erythema persisted. Her rheumatic symptoms were less severe and controlled by non-steroidal anti-inflammatory drugs, though there had been no regression of the radiological bone abnormalities.

\section{Comment}

Although the most familiar association of hypertrophic osteoarthropathy is with intrathoracic disease, it may also be hereditary or idiopathic. ${ }^{1}$ In addition, there is a well-recognised association with various extrathoracic disorders, especially a wide range of gastrointestinal diseases whose common feature is diarrhoea. ${ }^{12}$ Our patient's diarrhoea may be explained solely by purgative abuse, since it disappeared once she stopped the practice and there was no evidence of an underlying gastrointestinal disorder. The abnormality on barium follow-through examination was thought to be consistent with purgative abuse.

Silk $e t a l^{3}$ first described reversible finger-clubbing in a patient who ingested excessive quantities of purgatives, and the association has been noted by others, ${ }^{45}$ though in these instances the reversibility of clubbing could not be established because the patients persisted in taking purgatives. Interestingly these patients suffered from anorexia nervosa, and our patient's history suggested some features of this condition. We think that the presentation of clubbing with hypertrophic osteoarthropathy and purgative abuse is more than coincidental: there was no evidence of any other disease and, significantly, the patient's clubbing regressed and her symptoms improved after stopping the purgatives.

We thank Mr K M N Kunzru for referring the patient, and Dr G Sladen for helpful advice.

${ }^{1}$ Howell DS. Hypertrophic osteoarthropathy. In: McCarty DJ, ed. Arthritis and allied conditions. Philadelphia: Lea and Febiger, 1979:977-82.

2 Mendlowitz M. Clubbing and hypertrophic osteoarthropathy. Medicine. $1942 ; 21: 269-306$

${ }^{3}$ Silk DBA, Gibson JA, Murray CRH. Reversible finger clubbing in a case of purgative abuse. Gastroenterology 1975;68:790-4.

4 Prior J, White I. Tetany and clubbing in a patient who ingested large quantities of senna. Lancet 1978;ii:947.

${ }^{5}$ Malmquist J, Ericsson B, Hultén-Nosslin M-B, Jeppsson J-O, Ljungberg $O$. Finger clubbing and aspartylglucosamine excretion in a laxativeabusing patient. Postgrad Med $\mathcal{F} 1980 ; 56: 862-4$.

(Accepted 13 March 1981)

Department of Rheumatology, Guy's Hospital, London SE1 9RT

R D ARMSTRONG, MB, MRCP, senior registrar

A J CRISP, MB, MRCP, senior registrar

R GRAHAME, MD, FRCP, consultant rheumatologist

Department of Rheumatology, Wanstead Hospital, London E11

D L WOOLF, MRCP, consultant rheumatologist

\section{Primary biliary cirrhosis and focal glomerulonephritis}

Primary biliary cirrhosis is a progressive liver disease, and, though the aetiology is unknown, autoimmune mechanisms are probably implicated. Thomas $e t a^{1}$ suggested that the liver damage may be mediated by immune complex deposition, but evidence for this is not conclusive. Although immune complex deposition may mediate some of the diseases with which primary biliary cirrhosis is associated, to our knowledge only one case has been reported ${ }^{2}$ in which this has been clearly shown. We report a further case of primary biliary cirrhosis and immune complex disease and discuss briefly the importance of our findings. 